\title{
Inhalt
}

Vorwort zur 4. Auflage - V

\section{Abkürzungsverzeichnis - XVII}

1 Recht managen - 1

1.1 Ziele der Rechtsausbildung im Studium der BWL -1

1.2 Wege zur Zielerreichung - 1

1.3 Rechtsanwendung - 2

1.4 Erfolgreiche Prüfungsleistungen -4

2 Grundlagen Zivilrecht -6

$2.1 \quad$ Zivilrecht, Öffentliches Recht, Strafrecht

und andere Rechtsgebiete - 6

2.2 Die Rechtsquellen des Zivilrechts - 9

2.3 Das BGB -10

2.4 Der Inhalt des BGB -10

2.5 Von Babylon zum East River - $\mathbf{1 1}$

2.6 Die Person - 15

2.7 Zurechtfinden im BGB -19

3 Verträge - 21

$3.1 \quad$ Grundlagen -21

3.1.1 Begriff des Vertrags - 21

3.1.2 Verträge sind einzuhalten - 21

3.1.3 Der Grundsatz der Vertragsfreiheit - 22

3.1.4 Bindung durch Willensübereinstimmung - 24

3.1.5 Die Willenserklärung - 25

3.1.6 Der Grundsatz der Formfreiheit - 29

3.1.7 Vertrag und Gesetz - 30

3.1.8 Verpflichtungs- und Verfügungsgeschäft — 31

3.1.9 Ökonomische Begründung des Vertragsrechts - 32

$3.2 \quad$ Vorvertragliche Pflichten -33

3.2.1 Stellen Sie sich vor ... - 33

3.2.2 Vorvertragliche Pflichten im Überblick - 34

3.2.3 Ökonomische Bedeutung und Begründung - 35

3.2.4 Pflicht zum fairen Verhandeln - 35

3.2.5 Aufklärungspflichten - 37

3.2.6 Schutzpflichten - $\mathbf{3 8}$

3.2.7 Geheimhaltungspflichten -39 
3.2.8 Unbestellte Waren und Dienstleistungen - 39

3.2.9 Übungsfall $-\mathbf{4 0}$

3.2.10 Zusammenfassung - $\mathbf{4 1}$

3.3 Vertragsschluss $-\mathbf{4 1}$

3.3.1 Stellen Sie sich vor ... - 42

3.3.2 Ökonomische Bedeutung und Begründung - 42

3.3.3 Vertragsschluss durch Antrag und Annahme - 43

3.3.4 Erlöschen eines Antrags — 47

3.3.5 Annahme - $\mathbf{5 1}$

3.3.6 Kaufmännisches Bestätigungsschreiben - $\mathbf{5 3}$

3.3.7 Vertragsschluss ohne Angebot und Annahme? - 57

3.3.8 Übungsfall - $\mathbf{5 7}$

3.3.9 Zusammenfassung - $\mathbf{5 9}$

3.4 Besonderheiten bei Verbraucherverträgen $-\mathbf{5 9}$

3.4.1 Stellen Sie sich vor... 60

3.4.2 Ökonomische Bedeutung und Begründung - 61

3.4.3 Anwendungsbereich und Allgemeine Pflichten bei Verbraucherverträgen - $\mathbf{6 2}$

3.4.4 Außergeschäftsraumverträge und Fernabsatzverträge - 65

3.4.5 Rechtsfolgen der Außergeschäftsraum- und Fernabsatzverträge - 67

3.4.6 Widerruf -68

3.4.7 Rechtsfolgen des Widerrufs -70

3.4.8 Elektronischer Geschäftsverkehr — 71

3.4.9 Übungsfall -72

3.4.10 Zusammenfassung - 74

3.5 Vertragsschluss durch Stellvertreter -75

3.5.1 Stellen Sie sich vor ... 76

3.5.2 Ökonomische Bedeutung und Begründung — 77

3.5.3 Zulässigkeit der Stellvertretung - 78

3.5.4 Abgabe einer eigenen Willenserklärung -79

3.5.5 Handeln im Namen des Vertretenen — 79

3.5.6 Vertretungsmacht $-\mathbf{8 2}$

3.5.7 Rechtsfolgen wirksamer Stellvertretung - 90

3.5.8 Rechtsfolgen fehlender Vertretungsmacht $-\mathbf{9 0}$

3.5.9 Interessenkollisionen - 93

3.5.10 Übungsfall - 95

3.5.11 Zusammenfassung - 96

3.6 Unwirksamer Vertragsschluss - 97

3.6.1 Fehlende Geschäftsfähigkeit — 98

3.6.2 Anfechtung von Willenserklärungen — 101

3.6.3 Rechts- und sittenwidrige Rechtsgeschäfte - 112

3.6.4 Rückabwicklungen - $\mathbf{1 1 5}$ 
3.6.5 Zusammenfassung - $\mathbf{1 1 9}$

3.7 Allgemeine Geschäftsbedingungen — 119

3.7.1 Stellen Sie sich vor ... - $\mathbf{1 2 0}$

3.7.2 Ökonomische Bedeutung und Begründung - 120

3.7.3 Definition von AGB, § 305 Abs. 1 S. 1 BGB - 122

3.7.4 Wirksame Einbeziehung in den Vertrag, § 305 Abs. 2 BGB -125

3.7.5 Inhaltskontrolle $-\mathbf{1 2 8}$

3.7.6 Übungsfall 134

3.7.7 Zusammenfassung - 136

3.8 Erfüllung - 137

3.8.1 Stellen Sie sich vor ... 137

3.8.2 Ökonomische Bedeutung und Begründung — 137

3.8.3 Der richtige Schuldner - 138

3.8.4 Der richtige Gläubiger — 139

3.8.5 Die richtige Leistung - 139

3.8.6 Leistungsort -140

3.8.7 Leistungszeit $-\mathbf{1 4 2}$

3.8.8 Zahlung - 143

3.8.9 Aufrechnung und Verrechnung — 144

3.8.10 Übungsfall 144

3.8.11 Zusammenfassung - 146

3.9 Verjährung - 146

3.9.1 Stellen Sie sich vor ... 146

3.9.2 Ökonomische Bedeutung und Begründung — 147

3.9.3 Voraussetzungen -147

3.9.4 Rechtsfolgen - 149

3.9.5 Hemmung und Neubeginn $-\mathbf{1 5 0}$

3.9.6 Übungsfall — 151

3.9.7 Zusammenfassung - 152

$4 \quad$ Leistungsstörungen -153

4.1 Stellen Sie sich vor ... 153

4.2 Ökonomische Bedeutung und Begründung — 154

4.3 Grundlagen - 155

4.3.1 Leistungsverweigerungsrechte -155

4.3.2 Erfüllung - 157

4.3.3 Rücktritt 159

4.3.4 Schadensersatz -160

4.3.5 Aufwendungsersatz -163

4.3.6 Minderung -164

4.4 Unmöglichkeit - 165

4.4.1 Unmöglichkeit der Leistung — 166 
4.4.2 Rechtsfolgen -167

4.5 Verzug $\mathbf{1 6 8}$

4.5.1 Nichterbringung der möglichen Leistung zum richtigen Zeitpunkt - 169

4.5.2 Fälligkeit und Durchsetzbarkeit des Anspruchs des Gläubigers — 169

4.5.3 Mahnung des Gläubigers - $\mathbf{1 7 0}$

4.5.4 Vertretenmüssen der Verspätung — 172

4.5.5 Verzögerungsschaden beim Gläubiger — 173

4.5.6 Rechtsfolgen des Verzugs — 173

4.5.7 Verzug und Schadensersatz statt der Leistung - 174

4.5.8 Verzug und Rücktritt 176

4.6 Schlechtleistung - 178

4.7 Sonstige Pflichtverletzungen $-\mathbf{1 7 8}$

4.8 Übungsfall - 179

4.9 Zusammenfassung - 181

$5 \quad$ Kaufvertrag - 183

5.1 Stellen Sie sich vor ... $\mathbf{1 8 3}$

5.2 Ökonomische Bedeutung und Begründung — 184

5.3 Leistungspflichten der Parteien - 184

5.4 Gewährleistungsansprüche - $\mathbf{1 8 5}$

5.4.1 Sachmangel 185

5.4.2 Nacherfüllung - 190

5.4.3 Rücktritt -194

5.4.4 Minderung - 195

5.4.5 Schadensersatz -195

5.4.6 Aufwendungsersatz 198

5.4.7 Ausschluss und Beschränkung der Gewährleistungsrechte - 199

5.4.8 Verjährung der Mängelansprüche - 201

5.4.9 Rückgriff des Unternehmers - 203

5.5 Garantie - 204

5.5.1 Garantie und Gewährleistung - 204

5.5.2 Beschaffenheits- und Haltbarkeitsgarantie - 204

5.5.3 Inhalt der Garantie - 206

5.5.4 Garantie und Kulanz - 209

5.5.5 Wirtschaftliche Bedeutung der Garantie — 209

5.6 Übungsfall 210

5.7 Zusammenfassung -211

$6 \quad$ Werkvertrag - 213

6.1 Stellen Sie sich vor ... 214

6.2 Ökonomische Bedeutung und Begründung — 214 

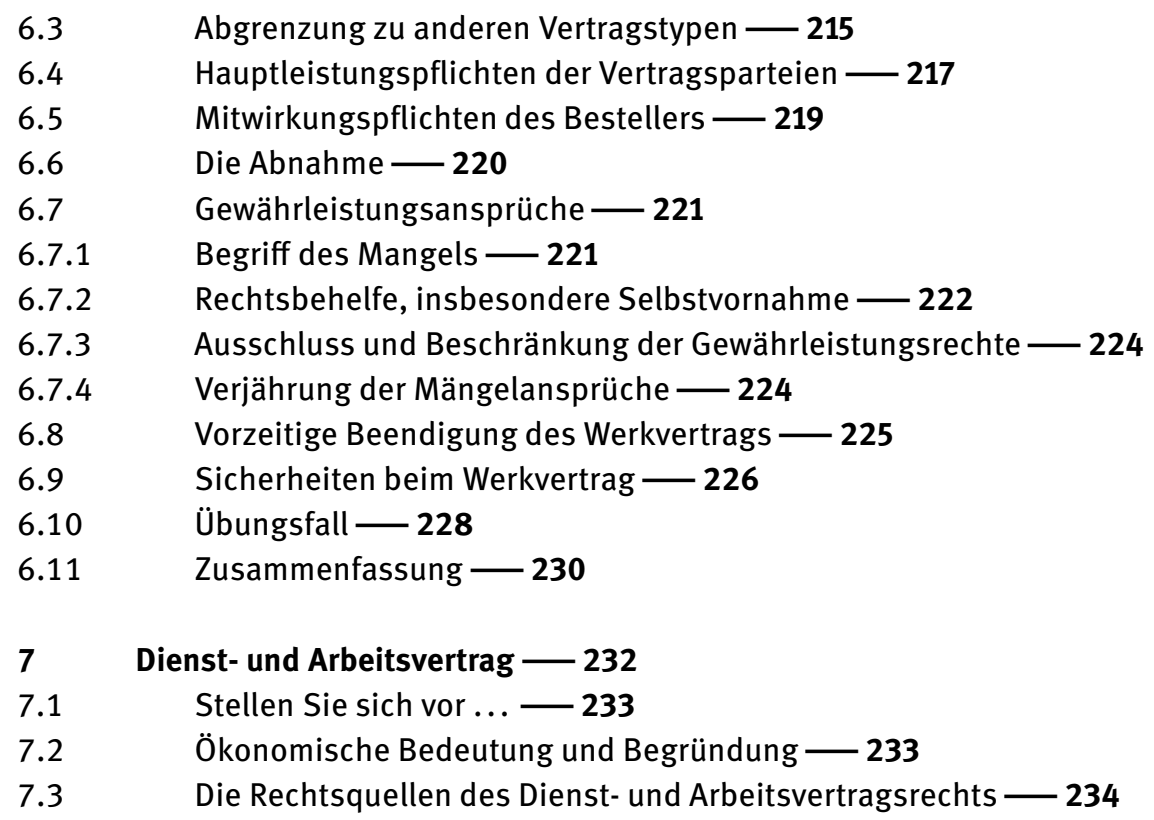

7.4 Abgrenzung zwischen Arbeitsvertrag und freiem Dienstvertrag — 237

7.5 Zustandekommen von Dienst- und Arbeitsvertrag - 240

7.5.1 Vertragsschluss $-\mathbf{2 4 0}$

7.5.2 Unwirksamkeitsgründe -242

7.6 Vertragspflichten -244

7.6.1 Pflichten beim Dienstvertrag — 244

7.6.2 Pflichten des Arbeitnehmers - 244

7.6.3 Pflichten des Arbeitgebers - 247

7.7 Leistungsstörungen $-\mathbf{2 5 0}$

7.8 Haftung für Schäden im Arbeitsverhältnis - 251

7.9 Beendigung von Dienst- und Arbeitsverhältnissen — 253

7.9.1 Arten der Beendigung - 253

7.9.2 Kündigungsschutzgesetz -255

$7.10 \quad$ Übungsfall 258

7.11 Zusammenfassung - $\mathbf{2 6 0}$

$8 \quad$ Handelsvertretervertrag -261

8.1 Stellen Sie sich vor ... - 261

8.2 Ökonomische Bedeutung und Begründung - 262

8.3 Pflichten von Handelsvertreter und Unternehmer - 262

8.4 Entgeltanspruch des Handelsvertreters - 263

8.5 Kündigung des Handelsvertretervertrags - 264

8.6 Der Ausgleichsanspruch — 265

8.7 Nachvertragliches Wettbewerbsverbot -266 
8.8 Übungsfall -267

8.9 Zusammenfassung $-\mathbf{2 6 8}$

9 Gesellschaftsverträge $-\mathbf{2 7 0}$

9.1 Stellen Sie sich vor ... - 271

9.2 Ökonomische Bedeutung und Begründung — 271

9.3 Vertraglicher Zusammenschluss zu einem gemeinsamen Zweck — 272

9.3.1 Entstehung von Personengesellschaften - 272

9.3.2 Entstehung von Kapitalgesellschaften $-\mathbf{2 7 5}$

9.4 Geschäftsführung und Vertretung - 280

9.4.1 Geschäftsführung und Vertretung bei GbR, OHG und KG - 280

9.4.2 Geschäftsführung und Vertretung der $\mathrm{GmbH}-\mathbf{2 8 2}$

9.5 Willensbildung der Gesellschafter - 283

9.5.1 GbR, OHG und KG - 283

9.5.2 $\mathrm{GmbH}-284$

9.6 Die Haftung der Gesellschafter für Gesellschaftsschulden - 285

9.6.1 Die Haftung in Kapitalgesellschaften - $\mathbf{2 8 5}$

9.6.2 Die Haftung in GbR, OHG und KG - 285

9.6.3 Die beschränkte Haftung des Kommanditisten — 286

9.6.4 Die GmbH \& Co. KG - 286

9.7 Ausscheiden und Beendigung — 287

9.7.1 Das freiwillige Ausscheiden eines Gesellschafters aus der Gesellschaft — 287

9.7.2 Das Ende der Gesellschaft - $\mathbf{2 8 8}$

9.8 Abgrenzung zu anderen Gesellschaftsformen - 289

9.9 Übungsfall und Zusammenfassung — 290

10 Darlehensverträge -293

10.1 Stellen Sie sich vor ... - 293

10.2 Ökonomische Bedeutung und Begründung — 294

10.3 Darlehen - 295

10.4 Verbraucherdarlehensverträge - 297

10.5 Verbundene Verträge — 299

10.6 Übungsfall $-\mathbf{3 0 0}$

10.7 Zusammenfassung - $\mathbf{3 0 1}$

11 Delikte - 303

$11.1 \quad$ Verschuldenshaftung - 303

11.1.1 Stellen Sie sich vor ... - 304

11.1.2 Ökonomische Bedeutung und Begründung - 304

11.1.3 Die Tatbestandsvoraussetzungen der Verschuldenshaftung im Überblick — 305 


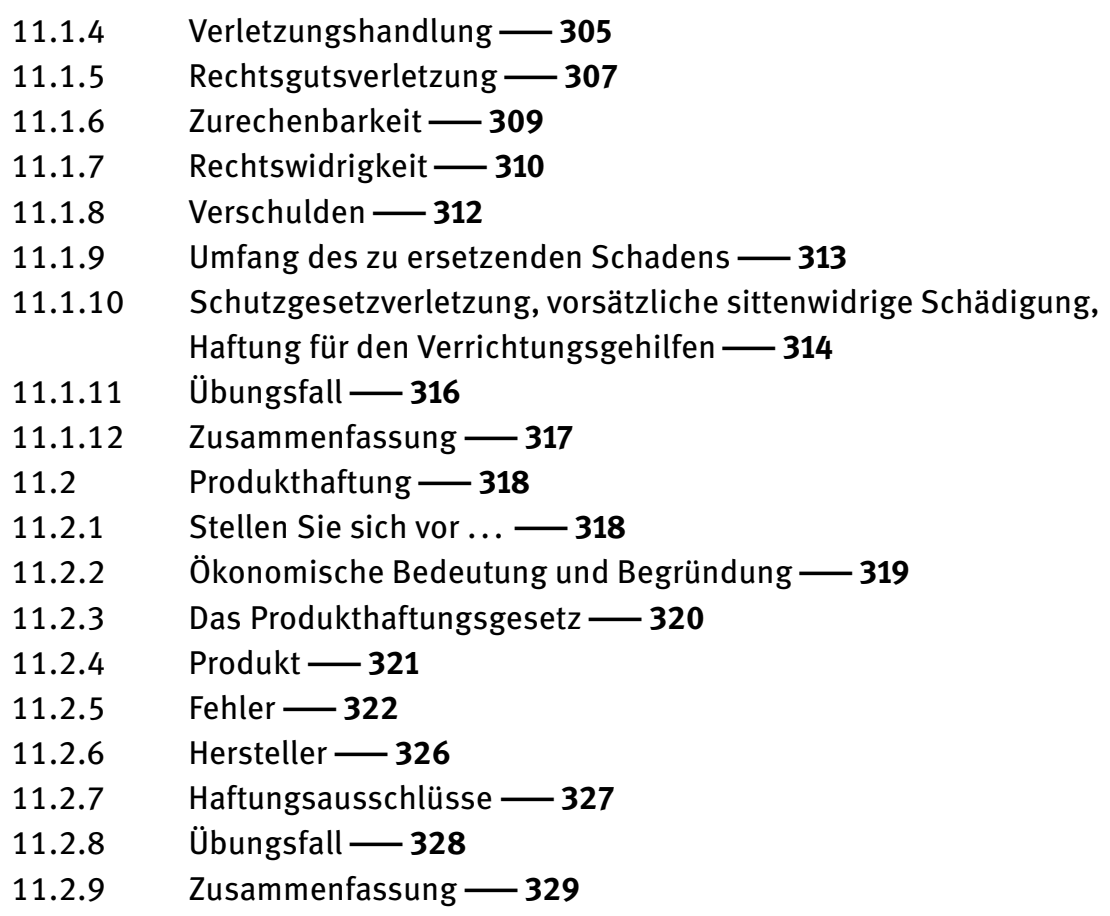

12 Eigentumserwerb an beweglichen Sachen - 330

12.1 Stellen Sie sich vor ... - $\mathbf{3 3 0}$

12.2 Ökonomische Bedeutung und Begründung des Eigentums — 331

12.3 Eigentum und Besitz -331

12.4 Besitz 332

12.4.1 Der Erwerb des unmittelbaren Besitzes - 332

12.4.2 Besitzbeendigung - 333

12.4.3 Unterscheidung zwischen unmittelbarem und mittelbarem Besitz - 333

12.4.4 Besitzschutzrechte - 334

$12.5 \quad$ Eigentumserwerb im Überblick — 335

$12.6 \quad$ Eigentumserwerb vom Berechtigten - 336

12.6.1 Übereignung nach § 929 S. 1 BGB - 336

12.6.2 Übergabe kurzer Hand gemäß §§ 929 S. 1, 929 S. 2 BGB - 338

12.6.3 Übereignung nach §§ 929 S. 1, 930 BGB - 339

12.6.4 Übereignung nach §§ 929 S. 1, 931 BGB - 339

12.7 Eigentumserwerb vom Nichtberechtigten - $\mathbf{3 4 0}$

12.7.1 §§ 929 S. 1, 932 Abs. 1 S. 1 BGB - 341

12.7.2 §§ 929 S. 1, 929 S. 2, 932 Abs. 1 S. 2 BGB - 341

12.7.3 $\S \S 929$ S. 1, 930, 933 BGB - 342

12.7.4 §§ 929 S. 1, 931, 934 BGB - 342 
12.7.5 Abhandengekommene Sachen - $\mathbf{3 4 3}$

12.7.6 Gutgläubiger Erwerb nach § 366 Abs. 1 HGB - 344

$12.8 \quad$ Gesetzlicher Eigentumserwerb - 344

12.8.1 Verbindung mit einem Grundstück - 344

12.8.2 Verbindung mit beweglichen Sachen - 345

12.8.3 Vermischung - 345

12.8.4 Verarbeitung - 346

12.9 Lösung des Eingangsfalls - 347

$12.10 \quad$ Zusammenfassung - $\mathbf{3 4 8}$

13 Erwerb von Forderungen - 349

13.1 Stellen Sie sich vor ... - $\mathbf{3 4 9}$

13.2 Ökonomische Bedeutung und Begründung - $\mathbf{3 5 0}$

13.3 Abtretung - $\mathbf{3 5 0}$

13.4 Sonderformen - 352

13.5 Leistung an den bisherigen Gläubiger — 353

13.6 Einwendungen des Schuldners - 354

13.7 Übungsfall - 356

$13.8 \quad$ Zusammenfassung $-\mathbf{3 5 7}$

$14 \quad$ Eigentumserwerb an Grundstücken - 358

14.1 Stellen Sie sich vor ... - $\mathbf{3 5 8}$

14.2 Ökonomische Bedeutung und Begründung - 359

14.3 Verpflichtung und Verfügung - 359

14.4 Notarielle Beurkundung des Verpflichtungsgeschäfts - 359

14.5 Auflassung und Eintragung im Grundbuch - 362

14.6 Lösung des Eingangsfalls - 363

$14.7 \quad$ Zusammenfassung - 364

15 Rechte an Grundstücken im Überblick - 365

15.1 Stellen Sie sich vor ... - 365

15.2 Ökonomische Bedeutung und Begründung - 365

$15.3 \quad$ Verpflichtung und Verfügung - 366

15.4 Grunddienstbarkeiten - 367

$15.5 \quad$ Nießbrauch - 367

15.6 Beschränkte persönliche Dienstbarkeit - 368

15.7 Erbbaurecht und Wohnungseigentum - 368

$15.8 \quad$ Grundschuld und Hypothek - 369

15.9 Vorkaufsrecht und Vormerkung - $\mathbf{3 7 0}$

15.10 Lösung des Eingangsfalls - $\mathbf{3 7 2}$

15.11 Zusammenfassung - $\mathbf{3 7 3}$ 
16 Kreditsicherheiten $-\mathbf{3 7 4}$

16.1 Stellen Sie sich vor ... - 374

16.2 Ökonomische Bedeutung und Begründung — 375

16.3 Bürgschaft - 376

16.3.1 Bürgschaftsvertrag - $\mathbf{3 7 8}$

16.3.2 Bürge und Gläubiger — 379

16.3.3 Bürge und Hauptschuldner $-\mathbf{3 8 0}$

16.3.4 Besonderer Schutz des Bürgen — 381

16.3.5 Ähnliche Sicherheiten - $\mathbf{3 8 3}$

$16.4 \quad$ Sicherungsabtretung - 384

16.4.1 Sicherungsabrede und Abtretung — 384

16.4.2 Erscheinungsformen - $\mathbf{3 8 5}$

16.4.3 Probleme der Sicherungsabtretung und ihre Konsequenzen — 386

16.5 Grundschuld und Hypothek - $\mathbf{3 8 7}$

16.6 Pfandrecht an beweglichen Sachen - $\mathbf{3 8 8}$

16.7 Sicherungsübereignung - 389

16.7.1 Die Sicherungsabrede - 389

16.7.2 Die Eigentumsübertragung — 391

16.7.3 Die Sicherungsübereignung eines Warenlagers - 392

16.7.4 Übersicherung - 392

16.7.5 Verwertung des Sicherungsguts - 393

16.7.6 Praktische Bedeutung — 393

16.8 Eigentumsvorbehalt -394

16.8.1 Der Eigentumsvorbehalt im Schuld- und Sachenrecht — 396

16.8.2 Formen des Eigentumsvorbehalts - 397

16.8.3 Kollision von Eigentumsvorbehalt und Globalzession - $\mathbf{4 0 0}$

16.8.4 Praktische Bedeutung des Eigentumsvorbehalts - $\mathbf{4 0 0}$

16.9 Lösung des Eingangsfalls - 401

16.10 Zusammenfassung - $\mathbf{4 0 2}$

Literaturhinweise -403

Autorinnen und Autoren -405

Stichwortverzeichnis -407 
